\title{
PROJETO DE VEÍCULO CICLOMOTOR COM ASSISTÊNCIA ELÉTRICA PARA TRANSPORTE URBANO
}

\author{
PROJECT OF A CYCLOMOTOR VEHICLE WITH ELECTRICAL ASSISTANCE FOR \\ URBAN TRANSPORT
}

\author{
Amanda Vieira Gomes Scandelai; Thaís Fernanda Menezes Barracar; César \\ Daltoé Berci.
}

Universidade do Oeste Paulista UNOESTE, Curso de Engenharia Elétrica, Presidente Prudente, SP

E-mail: cesarberci@unoeste.br

\begin{abstract}
RESUMO - A mobilidade urbana e o desenvolvimento sustentável, temas de grande impacto na qualidade de vida e bem estar social da população, se interrelacionam na questão do transporte motorizado, pois, o paradigma convencional para o deslocamento de pessoas e mercadorias, baseado em combustíveis fósseis, faz uso de uma fonte não renovável de energia e, além disso, é responsável pela emissão de gases nocivos, que contaminam o ar atmosférico, principalmente nos grandes centros urbanos de grande densidade demográfica, potencializando os danos por eles causados. Nesse contexto, pretende-se desenvolver com o projeto aqui proposto, uma alternativa baseada em um combustível renovável, aplicado a um meio de locomoção ágil e eficiente para trajetos urbanos, razão pela qual optou-se pela construção de uma bicicleta elétrica, que, associada a um baixo custo quando comparada as alternativas de transporte para a mesma aplicação, representa uma alternativa viável e economicamente acessível para o transporte urbano.

Palavras-chave: Bicicleta elétrica; bicicleta; autonomia.
\end{abstract}

ABSTRACT - Urban mobility and sustainable development, subjects of great impact on the life quality and population's social well-being, they are correlated to the motorized transport discussion, since the conventional paradigm for people and goods displacement are based on fossil fuels, makes use of a non-renewable source of energy and, in addition, is responsible for the emission of harmful gases, which contaminate the atmospheric air, mainly in the great urban centers with great population density, potentializing the damages caused by them. In this context, it is intended to develop, with the project proposed here, an alternative based on a renewable energy, applied to an agile and efficient means of transport for urban displacement, the reason why it was decided to build an electric bicycle, which, associated to a low cost when compared to others transport alternatives for the same application, represents a viable and economically accessible alternative for urban transport.

Keywords: Electric Bicycle; bicycle; autonomy 


\section{INTRODUÇÃO}

A história da Engenharia foi concebida com a história da humanidade e suas evoluções. Auxiliando o homem a resolver problemas do diaa-dia como a fabricação de artefatos para história do meio de locomoção ao qual destinamos o estudo deste trabalho. Alguns autores defendem Leonardo da Vinci como criador da primeira bicicleta, outros acreditam ser de Codex Atlanticus e ainda existem outros possíveis criadores.

Os modelos e as evoluções das bicicletas foram rápidas tornando-a mais segura e fácil de dirigir, abaixo segue figura dos marcos e evoluções.

Figura 1. Evolução da bicicleta

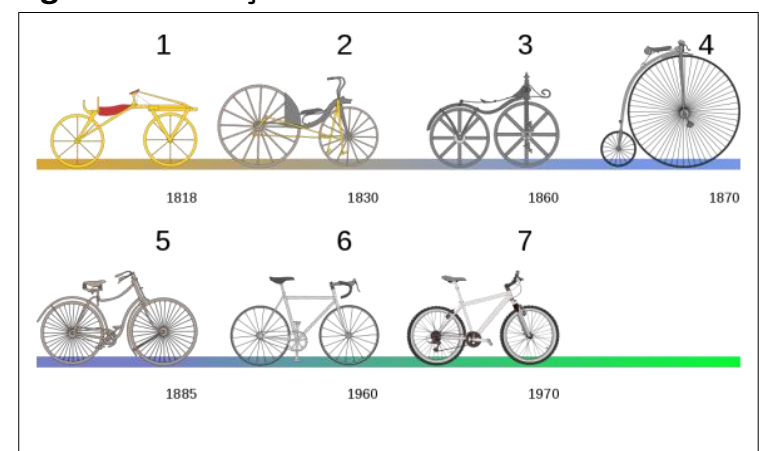

Fonte bibliográfica inválida especificada.

Segundo o a Federação do Comércio de Bens, Serviços e Turismo do Estado de São Paulo (FecomercioSP), a oferta de serviço no modal bicicleta humaniza o trânsito, reduz os congestionamentos e torna os deslocamentos saudáveis e sustentáveis.

De acordo com a Lei Federal 10.257/2001 (2015, p. 22, apud Soares) "A incorporação da bicicleta nas cidades deve fazer parte de uma política de mobilidade urbana que considere 0 desenvolvimento dos meios não motorizados de transporte, a fim de promover a inclusão social, a redução da poluição e a melhoria da saúde pública, contribuindo assim para a construção de cidades sustentáveis, o que é um direito estabelecido no Estatuto da Cidade".

Hoje em dia o número de automóveis e motocicletas vem aumentando nas cidades, consequentemente a emissão de gases poluentes ficou maior.

A ideia de construir uma bicicleta impulsionada por um motor elétrico surgiu em 1895 foi registrada a primeira patente para Ogden Bolton Jr, nos Estados Unidos da América, com modelo simples, uma bateria de $10 \mathrm{~V}$ e um motor que consumia 100 A (REVISTA BICICLETA, 2013).

O intuito da bicicleta é auxiliar no transporte podendo ser opcional pedalar. Atualmente, temos muitos modelos e estilos de bicicleta elétrica com autonomias diversas a fim de suprir cada necessidade do consumidor.

A bicicleta elétrica iniciou-se em 1895 e desde então tem um mercado crescente. No século XXI a bicicleta elétrica tomou impulso e suas fabricações no exterior foram de aproximadamente 400 mil unidades.

A principal vantagem da utilização da tração elétrica é a praticidade na mobilidade urbana sem esforço físico. Permiti utilizar um veículo pouco poluente reduzindo então a emissão de gases na atmosfera (CO2) e a poluição sonora.

Apesar da tração elétrica não emitir gases poluentes, após determinado tempo de uso a bateria utilizada como fonte tem uma vida útil, logo o mesmo deve ser descartado em local adequado, visto que se não for feito pode contaminar o solo e lençóis freáticos.

O modelo que iremos desenvolver será uma bicicleta de 18 marchas de aro 26, será considerado um motor elétrico de corrente contínua (DC) de $880 \mathrm{~W}$ de potência, uma bateria selada de 12 V e 12 Ah. Serão analisados cada componente da bicicleta objetivando melhor relação custo benefício.

\section{OBJETIVOS ESPERADOS}

\subsection{Objetivo Geral}

Construir uma bicicleta que alcance curtas e médias distâncias com uma velocidade média de $25 \mathrm{~km} / \mathrm{h}$. Destinada a trajetos urbanos curtos, como: locomoção para ir ao trabalho, ir à escola e lazer.

Pretende-se também reduzir o custo da bicicleta elétrica para ser acessível a grande parte da população.

\subsection{Objetivos Específicos}

- Elaborar um projeto de baixo custo;

- Analisar o mercado de bicicletas elétricas;

- Desenvolver um projeto para a adaptação de uma bicicleta comum em uma bicicleta elétrica.

O público alvo do nosso projeto serão homens e mulheres de 15 a 45 anos de classe média baixa az média alta. A utilização deste 
meio de locomoção será destinada ao trabalho, escola e lazer.

\section{PROJETO}

Para os cálculos de desempenho utilizamos os seguintes dados que foram retirados das especificações técnicas dos produtos e informações que obtivemos a partir de cálculos e análises.

Tabela 1. Dados Técnicos

\begin{tabular}{|l|c|c|c|}
\hline Variável & Sym & Val. & Uni \\
\hline Torque máximo & $\mathrm{Tm}$ & 0,8 & $\mathrm{Nm}$ \\
\hline Velocidade do motor & $\mathrm{Wm}$ & 1000 & $\mathrm{RPM}$ \\
\hline $\begin{array}{l}\text { Diâmetro externo do } \\
\text { pneu }\end{array}$ & $\Phi$ & 56 & $\mathrm{Cm}$ \\
\hline N. de dentes do pinhão & $\mathrm{Np}$ & 16 & \\
\hline N. de dentes da coroa & $\mathrm{Nc}$ & 48 & \\
\hline Perda mecânica & $\mathrm{Ml}$ & \multicolumn{2}{|c|}{ Desconsiderado } \\
\hline Peso do conjunto & $\mathrm{M}$ & 120 & $\mathrm{Kg}$ \\
\hline Velocidade de cruzeiro & $\mathrm{Vc}$ & 28,6 & $\mathrm{~km} / \mathrm{h}$ \\
\hline
\end{tabular}

Fonte bibliográfica inválida especificada.

O sistema de relação de transmissão adota engrenagens com diferentes números de dentes para multiplicar ou reduzir a força do motor. Foi calculada a partir do número de dentes da coroa em relação ao número de dentes do pinhão, conforme demonstrado na fórmula abaixo:

$$
\begin{aligned}
K & =\frac{n C}{n P} \\
K & =3,00
\end{aligned}
$$

Onde: $K$ a relação de transmissão; $n C$ número de dentes da coroa; $n P$ número de dentes do pinhão.

O módulo do torque máximo é calculado a partir da relação de transmissão e do torque do motor, conforme demonstrado na fórmula abaixo:

$$
\begin{gathered}
\left\|\vec{T}_{\text {max }}\right\|=\frac{\left(K \cdot T_{m}\right)}{g} \\
\left\|\vec{T}_{\text {max }}\right\|=2,4 \mathrm{~N} \cdot \mathrm{m}
\end{gathered}
$$

Sendo: $\vec{T}_{\max }$ torque máximo com sua unidade de medida em $N \cdot m ; K$ relação de transmissão; $T_{m}$ módulo do torque do motor.

A velocidade angular nominal foi calculada a partir da velocidade do motor e da relação de transmissão, conforme demonstrado na fórmula abaixo:

$$
\begin{gathered}
\omega=\frac{W_{m}}{K} \\
\omega=333 \mathrm{rpm}
\end{gathered}
$$

Sendo: $\omega$ velocidade angular nominal com sua unidade em rpm; $W_{m}$ velocidade do motor; $K$ relação de transmissão.

O módulo da velocidade máxima foi calculado a partir da velocidade angular nominal e do diâmetro do pneu, convertendo esta equação para $\frac{K m}{h}$.

$$
\begin{gathered}
\left\|\vec{V}_{\text {max }}\right\|=\frac{\omega \cdot 2 \pi \cdot \phi \cdot 3,6}{60 \cdot 200} \\
\left\|\vec{V}_{\text {max }}\right\|=\frac{333 \cdot 2 \pi \cdot 56 \cdot 3,6}{60 \cdot 200} \\
\left\|\vec{V}_{\text {max }}\right\|=35,2 \mathrm{~km} / \mathrm{h}
\end{gathered}
$$

Sendo: $\vec{V}_{\text {max }}$ velocidade com sua unidade de medida $\mathrm{km} / \mathrm{h} ; \omega$ velocidade angular nominal; $\phi$ diâmetro da roda.

O módulo da força que a roda faz sobre a superfície de contato foi calculado a partir aceleração da gravidade e do módulo do torque máximo em relação ao diâmetro do pneu, conforme demonstrado na fórmula abaixo, onde estamos considerando que $\vec{F}$ é uma grandeza vetorial que possui um módulo e uma orientação, e seu módulo é dado por $\|\vec{F}\|$.

Basicamente o torque $T_{\text {max }}$ é a força $\|\vec{F}\|$ multiplicada pelo raio $R$.

$$
\begin{gathered}
\|\vec{F}\|=\frac{T_{\text {max }}}{R} \\
\|\vec{F}\|=84 N
\end{gathered}
$$

Onde: $F$ força com sua unidade de medida em Newton $(N) ; T_{\max }$ torque máximo; $\phi$ Diâmetro externo do pneu; $R$ Raio da roda.

O módulo da aceleração é obtido da seguinte equação:

$$
\vec{a}(m)=\frac{\vec{F}}{m}
$$

Analogamente, prova-se que, dentro desse contexto, quanto menor a massa maior será o módulo da aceleração, ou seja, $\lim _{m \rightarrow 0} \vec{a}(m)=$ $\lim _{m \rightarrow 0} \frac{\vec{F}}{m}=+\infty$, conforme demonstrado na figura 2 . 
Figura 2. Aceleração em função da massa

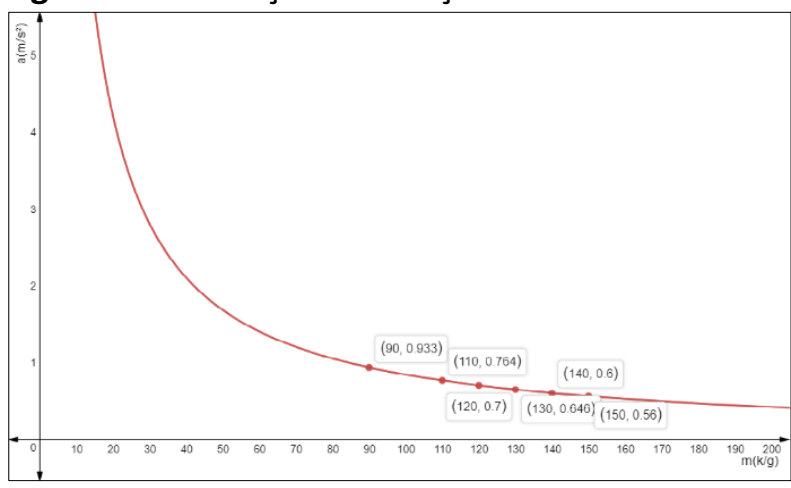

Fonte bibliográfica inválida especificada.

A fim de determinar a velocidade de cruzeiro da bicicleta, utilizaremos a velocidade nominal do motor em conjunto com a relação de transmissão e a dimensão da roda, conforme segue:

$$
v_{c}=\omega_{r} \cdot R
$$

Para isso precisaremos calcular $\omega_{r}$ :

$$
\omega_{\mathrm{r}}=\frac{\omega_{\mathrm{n}}}{\mathrm{k}}
$$

Sendo: $v_{c}$ velocidade de cruzeiro; $\omega_{r}$ velocidade angular da roda; $\omega_{n}$ velocidade angular nominal; $k$ relação de transmissão.

Seja $\omega_{n}$ velocidade angular nominal calculada a partir da razão entre deslocamento angular pelo período gasto para dar uma volta completa na circunferência. Conforme a fórmula abaixo:

$$
\omega_{n}=\frac{2 \pi}{T}=\frac{2 \pi}{\frac{60}{R o t}}=\frac{2 \pi}{\frac{60}{1000}}=\frac{2 \pi}{0,06}=104 \frac{\mathrm{rad}}{\mathrm{s}}
$$

Portanto, substituindo o valor da velocidade angular nominal na fórmula obteremos a velocidade angular da roda.

$$
\omega_{\mathrm{r}}=\frac{\omega_{\mathrm{n}}}{\mathrm{k}}=\frac{104}{3,00}=34,6 \frac{\mathrm{rad}}{\mathrm{s}}
$$

Logo, a velocidade de cruzeiro será calculada a partir do produto da velocidade angular da roda e raio da roda

$$
v_{c}=\omega_{r} \cdot R=7,9 \frac{\mathrm{m}}{\mathrm{s}}=28,6 \frac{\mathrm{km}}{\mathrm{h}}
$$

Sendo: $v_{c}$ velocidade de cruzeiro; $R$ raio da roda.

Para sabermos o tempo $t$, com sua unidade de medida em segundos, em que a bicicleta levará para atingir sua velocidade de cruzeiro $V c$, calculamos:

$$
t=\frac{\frac{V c}{3,6}}{\|\vec{a}\|}
$$

Sendo: $t$ tempo com sua unidade de medida segundos; $m$ massa; $v_{c}$ velocidade de cruzeiro; $\|\vec{a}\|$ módulo da aceleração.
A potência mecânica é obtida da seguinte equação:

$$
\begin{aligned}
P_{n} & =\frac{m \cdot{\frac{v_{c}}{3,6}}^{2}}{2 \cdot t} \\
P_{n} & =326 \mathrm{~W}
\end{aligned}
$$

Sendo: $P_{n}$ potencia mecânica com sua unidade de medida Watts; $\mathrm{m}$ massa; $v_{c}$ velocidade de cruzeiro; $\mathrm{t}$ tempo em segundos $(s)$.

Consideremos agora, uma situação onde é necessário transitar por uma via inclinada. Para que haja movimento o módulo da força motora deve satisfazer a seguinte condição:

$$
\vec{F}_{m}>\vec{P} \sin \theta \cdot \hat{\imath}
$$

Para sabermos qual o ângulo máximo que a bicicleta conseguirá subir calculamos:

$$
\begin{gathered}
\theta=\sin ^{-1} \frac{\|\vec{F}\|}{\|\vec{P}\|} \\
\vec{P}=M \cdot \overrightarrow{\mathrm{g}} . \\
\theta=4^{\circ}
\end{gathered}
$$

Figura 3. Inclinação máxima

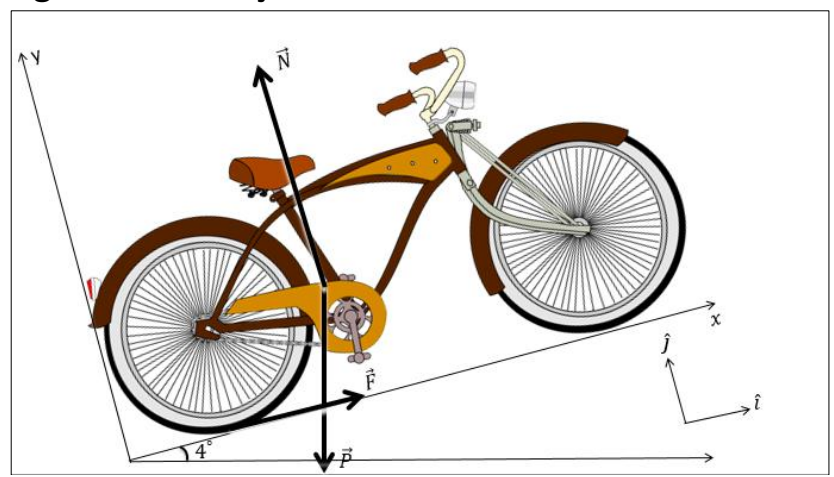

Fonte bibliográfica inválida especificada.

Sendo: $\theta$ o ângulo máximo que a bicicleta conseguirá subir uma determinada inclinação com sua unidade de medida em graus; $F$ módulo da força do motor; $M$ a massa da bicicleta com o ciclista; $g$ aceleração da gravidade.

No quadro abaixo demonstramos uma relação de força com a inclinação: 
Tabela 2. Relação de Força com a Inclinação

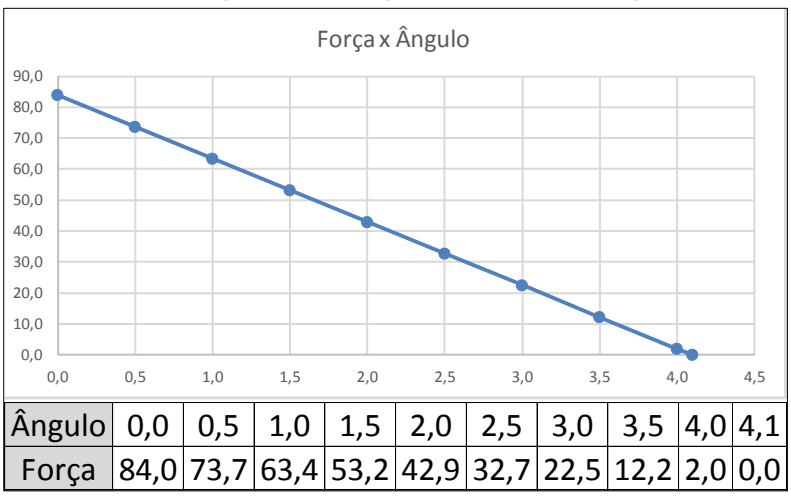

Fonte bibliográfica inválida especificada.

Para os cálculos em relação à bateria utilizamos os seguintes dados, retirados das especificações técnicas dos produtos e informações que obtivemos a partir de cálculos e análises.

Tabela 3. Dados Técnicos

\begin{tabular}{|l|c|c|c|}
\hline \multicolumn{1}{|c|}{ Variável } & Sym. & Val. & Uni \\
\hline Tensão das baterias & $\mathrm{Vb}$ & 12 & $\mathrm{~V}$ \\
\hline Capacidade de corrente & $\mathrm{Ib}$ & 12 & $\mathrm{Ah}$ \\
\hline Potência do motor & $\mathrm{P}$ & 250 & $\mathrm{~W}$ \\
\hline
\end{tabular}

Fonte bibliográfica inválida especificada.

A energia total disponível na bateria é dada por:

$$
E_{b}=V_{b} \cdot I_{b}=518.400 \mathrm{~J}
$$

Utilizando-se agora dos dados do motor, é possível calcular o intervalo de tempo mínimo de duração da bateria, o que se faz como segue:

$$
E=P \cdot \Delta t
$$

$\Delta t=34,5$ minutos

Preservando-se as unidades, é possível calcular a distância mínima que pode ser percorrida com uma carga, ou, popularmente chamada de autonomia, utilizando a velocidade de cruzeiro e o tempo de duração da bateria.

$$
\begin{gathered}
\Delta x=v_{c} \cdot \Delta t \\
\Delta x \approx 16,5 \mathrm{Km}
\end{gathered}
$$

\section{TESTES E VALIDAÇÃO}

Com a finalidade de validar o projeto, foi montado um protótipo inicial da bicicleta.
Figura 4. Teste da Bicicleta

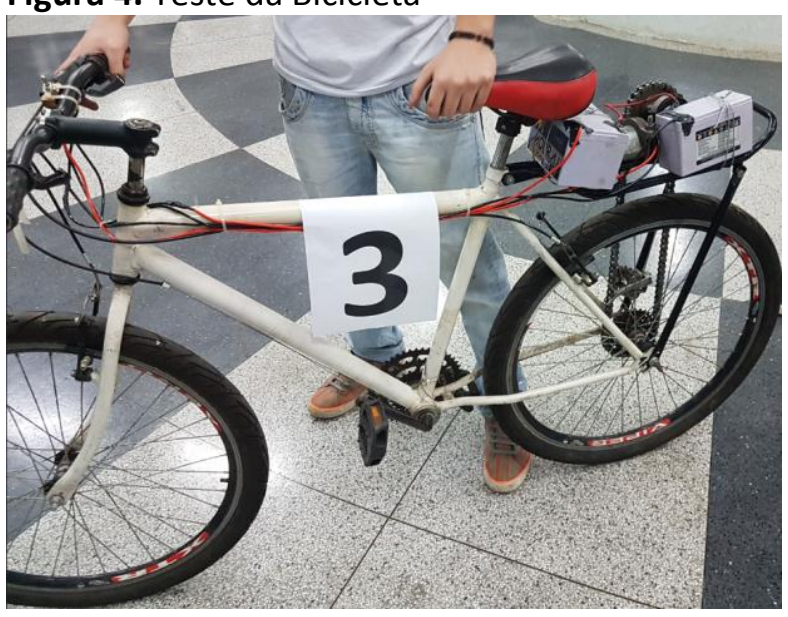

Os testes foram realizados percorrendo uma distância de 25 metros, conforme figura 5, procedimento que foi repetido por cinco vezes.

A partir desses testes, obtivemos Uma coleção de valores de tempo, sendo um para cada realização do experimento.

Figura 5. Teste da Bicicleta

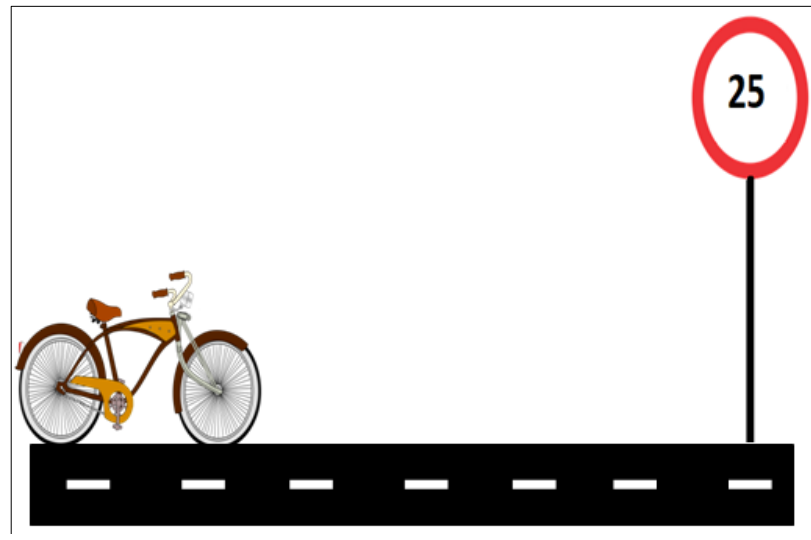

Fonte bibliográfica inválida especificada.

Os valores de tempo obtidos foram utilizados para estimar a aceleração da bicicleta, a qual, apoiada pela continuidade do torque do motor DC em relação a sua rotação, espera-se ser constante em relação à velocidade.

Utilizando uma aproximação através do método dos mínimos quadrados, foi obtido o seguinte valor para a aceleração:

$$
a=0,672 \mathrm{~m} / \mathrm{s}^{2}
$$

Esse resultado é consistente com os dados levantados no projeto.

\section{LEVANTAMENTO DOS CUSTOS}

Os materiais e valores de serviço que serão utilizados estão listados na tabela abaixo: 
Tabela 4. Levantamento dos Custos

\begin{tabular}{|l|l|l|}
\hline Descrição & Produto & Serviço \\
\hline Bicicleta & 500,00 & 0,00 \\
\hline Motor DC & 200,00 & 0,00 \\
\hline Bateria & 80,00 & 0,00 \\
\hline Componentes elétricos & 150,00 & 0,00 \\
\hline $\begin{array}{l}\text { Componentes } \\
\text { complementares }\end{array}$ & 110,00 & 0,00 \\
\hline Mão de Obra & 0,00 & 300,00 \\
\hline Subtotal & $1.040,00$ & 300,00 \\
\hline Total & & $1.340,00$ \\
\hline
\end{tabular}

Fonte bibliográfica inválida especificada.

\section{CONCLUSÃO}

Através deste projeto pode-se demonstrar que a utilização de uma bicicleta com tração elétrica para o dia a dia é de grande importância, analisando o ganho que o meio ambiente tem pela diminuição de gases poluentes na atmosfera, para a saúde física mental do ciclista e a redução de custos com manutenção de carro, combustível entre outros gastos que um veículo proporciona.

Foram identificados através dos cálculos e análise do custo benefício quais equipamentos deveriam ser utilizados para atender os pré-requisitos que havíamos estipulado.

Com os cálculos foi possível validar que o ciclista com um Peso médio de $80 \mathrm{~kg}$ (peso total do conjunto $120 \mathrm{~kg}$ ) atingira uma velocidade de $28,6 \mathrm{~km} / \mathrm{h}$ com aceleração de $0,7 \mathrm{~m} / \mathrm{s}^{2}$ em 11,36 segundos, com a força do motor a bicicleta poderá subir uma inclinação de até $4^{\circ}$, a autonomia da bateria é de 34,5 minutos sendo que a distância máxima percorrida com um ciclo de bateria será de $16 \mathrm{~km}$.

A expectativa da bicicleta foi atendida dentro dos parâmetros propostos. Esse projeto mostrou-se economicamente viável, somando todos os componentes e serviços necessários o valor final foi de $\mathrm{R} \$ 1.340,00$, o preço de venda sugerido é de uma margem de $20 \%$ de lucro aproximadamente $\mathrm{R} \$ 1.600,00$.

Com ciclo de bateria da bicicleta consegue alcançar $16,6 \mathrm{Km}$ e seu consumo para carregar é 144 Wh com tempo de carga completa sendo de $3 \mathrm{~h}$. Supondo o preço do Wh $\mathrm{R} \$ 0,62$ $\mathrm{kWh}$, calculou-se um gasto de $\mathrm{R} \$ 0,30$ por ciclo.
Com um automóvel de autonomia $8 \mathrm{~km}$ com 1 litro e o preço atual do etanol $\mathrm{R} \$ 2,89$, percorrendo a mesma distância determinou um gasto de $\mathrm{R} \$ 5,78$.

Portanto, a bicicleta mostrou-se economicamente viável, havendo uma economia de $R \$ 5,48$.

Em uma simulação, uma pessoa que utiliza um automóvel para locomoção em um percurso diário de $16 \mathrm{~km}$, gastaria $\mathrm{R} \$ 173,40$ mensal, se o mesmo optar pela bicicleta elétrica gastaria em um mês $\mathrm{R} \$$ 9,00. Com essa simulação, houve uma economia em seus gastos de $\mathrm{R} \$ 164,40$ ao mês

\section{REFERÊNCIAS}

Aguias do Asfalto. (5 de 04 de 2009). http://www.aguiasdoasfaltosp.com.br/dicas/mot ores_torque_potencia.htm. Fonte: Aguias do Asfalto:

http://www.aguiasdoasfaltosp.com.br/dicas/mot ores_torque_potencia.htm

Autoria Própria, A. (02 de 12 de 2018). Presidente Prudente, São Paulo, Brasil.

Conceito.de. (1 de 11 de 2014). Conceito.de.

Fonte: Conceito.de:

https://conceito.de/potencia-mecanica

Conselho de Desenvolvimento Local da Federação do Comércio de Bens, Serviços e Turismo do Estado de São Paulo (FecomercioSP). (18 de 05 de 2016). FecomercioSP. Acesso em 30 de 09 de 2017, disponível em FecomercioSP: http://www.fecomercio.com.br/noticia/a-hora-ea-vez-de-lucrar-com-as-bicicletas

eCycle. (01 de 09 de 2014). eCycle. Acesso em 30 de 09 de 2017, disponível em eCycle:

https://www.ecycle.com.br/component/content/ article/41-pegue-leve/2849-bikes-eletricashistoria-mercado-expansao-motorcaracteristicas-vantagens-praticidade-pedalartrabalho-limitacao-fisica-custo-economiaacelerador-integrado-separado-solar-movimentobem

Globo.com. (21 de 9 de 2015). Fonte: Globo.com: http://educacao.globo.com/fisica/assunto/mecan ica/movimentos-circulares.html 
O Portal da Bicicleta. (16 de 01 de 2013). O Portal da Bicicleta. Acesso em 30 de 09 de 2017, disponível em Bicicleta:

http://www.revistabicicleta.com.br/bicicleta.php ?especial_bicicleta_eletrica\&id=2351

Soares, R. D. (01 de 11 de 2015). USPNET. Acesso em 30 de 09 de 2017, disponível em USPNET:

http://myrtus.uspnet.usp.br/celacc/sites/default/ files/media/tcc/versao_final_pdft.pdf

Whittell, G. (1993). Lambada Country: A Ride Across Eastern Europe.

Wikipedia. (14 de 10 de 2017). Fonte: Wikipedia: https://pt.wikipedia.org/wiki/Acelera\%C3\%A7\%C 3\%A3०

Wikipedia. (20 de 10 de 2017). Wikipedia. Fonte: Wikipedia: https://pt.wikipedia.org/wiki/Bicicleta 